\title{
Proportion of acute ischaemic strokes attributable to a cardiac aetiology in an unselected young patient population: A single centre experience
}

\author{
Authors: Rahul Bahl, ${ }^{\mathrm{A}}$ Magdalena Kubiak, ${ }^{\mathrm{A}}$ Syarah Yusoff, ${ }^{\mathrm{A}}$ Hsiu Tung, ${ }^{\mathrm{A}}$ James Stirrup, ${ }^{\mathrm{B}}$ André van Wyk, \\ Kiruba Nagaratnam ${ }^{B}$ and Neil Ruparelia ${ }^{D}$
}

The incidence of acute ischaemic stroke in young patients is increasing and identifying the underlying cause is critically important with regards to their optimal management. The true proportion of cardiac causes of stroke in young patients is poorly defined. We aimed to determine the proportion of strokes attributable to cardiac causes in an unselected, consecutive cohort of young patients. We used the database of a large stroke service to identify patients aged $\leq 55$ years presenting with stroke between 01 January 2015 and 31 December 2017. We reviewed their clinical notes and investigations and then categorised patients by the cause of their stroke.

We screened 202 cases, and excluded 35, resulting in a study population of 167 patients; $24.0 \%(40 / 167)$ had a cardiac cause of stroke including $9.6 \%(16 / 167)$ had patent foramen ovale, 9.0\% (15/167) had intracardiac source of embolus and $5.4 \%(9 / 167)$ had atrial fibrillation; $50.8 \%$ (85/167) had other more likely causes; and $25.1 \%(42 / 167)$ had no clear underlying cause.

A high proportion (24\%) of strokes in young patients are secondary to a cardiac cause. Thorough investigation in these patients is warranted and requires close interdisciplinary links between cardiologists and stroke physicians to ensure optimal management.

KEYWORDS: Stroke, atrial fibrillation, patent foramen ovale, aetiology, cardiology

DOI: 10.7861/clinmed.2019-0353

\section{Introduction}

The incidence of ischaemic stroke in young patients is increasing and is associated with significant morbidity, mortality and socioeconomic

Authors: ${ }^{\text {A }}$ core trainee, Royal Berkshire Hospital, Reading, UK; ${ }^{B}$ consultant cardiologist, Royal Berkshire Hospital, Reading, UK; Consultant stroke physician, Royal Berkshire Hospital, Reading,

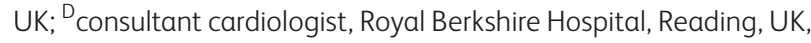
Hammersmith Hospital, London, UK and Imperial College London, London, UK cost. Investigation of the underlying cause of stroke in these patients is critical to their overall management to guide pharmacotherapy, reduce the risk of recurrence and improve long-term outcomes.

Cardiac causes of stroke include atrial fibrillation or flutter (AF), patent foramen ovale (PFO) or an intracardiac source of embolus relating to valvular heart disease or impaired ventricular function. In addition to guiding optimal medical therapy (such as formal anticoagulation), this area has recently gained greater interest with randomised controlled trial evidence showing a significant reduction in recurrent ischaemic events following percutaneous PFO closure in young patients with cryptogenic ischaemic stroke. ${ }^{2-4}$

The identification of underlying cardiac pathology in these patients requires extensive investigation and therefore has implications for cardiology resources. Outside of the clinical trial setting the true incidence of cardiac causes of stroke is not well defined in younger patients. We aimed to determine the proportion of acute ischaemic strokes attributable to cardiac causes in this patient group in an unselected consecutive cohort.

\section{Methods}

We used the local database of a large UK stroke service to identify patients aged $\leq 55$ years presenting with stroke between 01 January 2015 and 31 December 2017. A stroke was diagnosed on the basis of clinical examination with a focal neurological deficit by a stroke physician, usually supported by imaging (computed tomography (CT) and/or magnetic resonance imaging (MRI)). As part of a national audit of stroke activity, all patients diagnosed with a stroke are routinely entered into the database. We reviewed clinical letters, imaging reports, cardiac investigation reports and laboratory reports and inputted the relevant details into a dedicated predefined anonymised database. Exclusion criteria included patients presenting with haemorrhagic stroke, patients where the clinical impression was not a stroke, patients who were lost to followup and therefore not investigated, and patients who were older than 55 years. We counted duplicate presentations with stroke as one episode. We categorised cause of stroke according to the Trial of Acute Stroke Treatment (TOAST) criteria. ${ }^{5}$ We defined cardiac causes of stroke as AF, PFO and intracardiac source of embolus. We defined intracardiac source of embolus as valvular heart disease (endocarditis or prosthetic valve), visualised intracardiac thrombus or heart failure. Where there was more than one cause, we selected the most likely cause by consensus of three reviewers. 


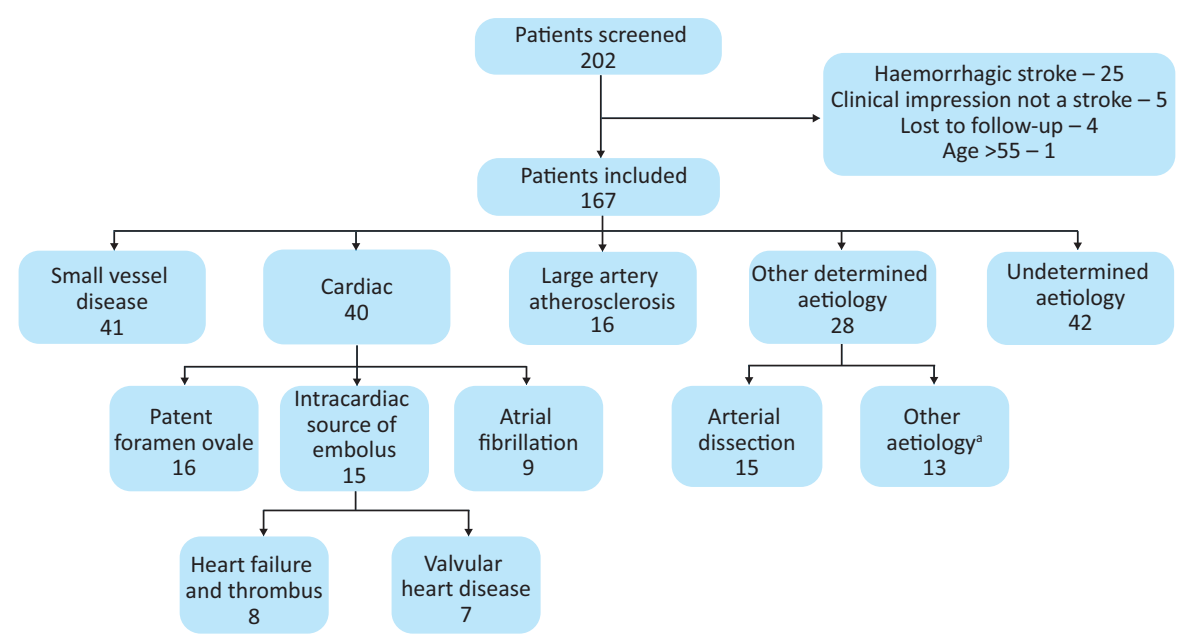

Fig 1. Flowchart showing patient inclusion and categorisation by cause of stroke. ${ }^{a}=$ including malignancy, prothrombotic state, metabolic and genetic disease.
Given the current randomised controlled trial evidence for PFO closure in patients with cryptogenic stroke, PFO was defined as the cause of stroke only if there was no other cause. Patients where a clear cause of stroke could not be accurately defined on the basis of available information were categorised as a stroke of undetermined cause.

Data are presented as absolute numbers and proportions of the total included population of young patients presenting with ischaemic stroke for each TOAST category (cardiac, small vessel disease, large artery atherosclerosis, other determined aetiology and undetermined aetiology) and for significant subcategories.

\section{Results}

During the study period, 202 patients were identified as having suffered a stroke. Thirty-five patients were excluded: 25 with haemorrhagic stroke, five where the clinical impression was not a stroke, four who were lost to follow-up and not investigated and one who was aged greater than 55 years. This left a final study population of 167 patients (Fig 1).

Of the patients included, ages ranged from 19 to 55 years (median age 49 years); $32.3 \%$ (54/167) were female; $36.5 \%$ (61/167) had a history of hypertension; $14.4 \%$ (24/167) had a history of diabetes mellitus and $12.6 \%$ (21/167) had a history of prior stroke or transient ischaemic attack. All included patients had brain imaging performed with CT and/or MRI and 90.4\% (151/167) of patients had vascular imaging performed with ultrasound Doppler, CT angiography (CTA) or magnetic resonance angiography (MRA); $64.7 \%(108 / 167)$ of patients had ambulatory electrocardiography (ECG) monitoring; 65.3\% (109/167) had transthoracic echocardiography (TTE) performed and 23.4\% (39/167) had bubble contrast echocardiography performed (Table 1).

Average duration of ECG monitoring was 68.4 hours and AF was detected on $1.8 \%$ (2/109) of recordings. Eight patients had a known prior diagnosis of AF and so were not investigated with ambulatory ECG monitoring and four had a new diagnosis AF discovered on 12-lead ECG. A positive result was found on $19.3 \%(21 / 109)$ of TTEs performed. PFO was discovered on the basis of $41.0 \%(16 / 39)$ of bubble contrast echocardiography performed.

Cardiac cause of stroke were identified in 24.0\% (40/167) including $9.6 \%(16 / 167)$ with PFO, 9.0\% (15/167) with intracardiac source of embolus and 5.4\% (9/167) with AF; 50.9\% (85/167) were thought to have other causes: $24.6 \%$ (41/167) with small vessel disease; $9.6 \%(16 / 167)$ with large artery atherosclerosis; and $16.8 \%$ (28/167) with other determined aetiology including 9.0\% (15/167) with arterial dissection, $2.4 \%$ (4/167) due to cancer and 1.8\% (3/167) due to antiphospholipid syndrome; other rare causes included drug use, genetic disorders and lysosomal and mitochondrial disorders; and 25.1\% (42/167) presented with a stroke but with no clear underlying cause found (Fig 2).

Table 1. Investigations performed on included

\section{patients}

$\begin{array}{ll}\text { Investigation } & \begin{array}{l}\text { Number performed, } \\ \text { total } \mathbf{n = 1 6 7 ,} \mathbf{n}(\%)\end{array} \\ \text { Brain imaging } & 160(95.8) \\ \text { CT } & 123(73.7) \\ \text { MRI } & 167(100.0) \\ \text { CT or MRI } & \\ \text { Ambulatory ECG } & 108(64.7) \\ \text { Holter or R test } & \\ \text { Vascular imaging } & 34(20.4) \\ \text { US Doppler } & 39(23.4) \\ \text { CTA } & 87(52.1) \\ \text { MRA } & 151(90.4) \\ \text { US or CT or MRI } & \\ \text { Cardiac imaging } & 109(65.3) \\ \text { Transthoracic echocardiography } & 39(23.4) \\ \text { Bubble echocardiography } & 7(4.2) \\ \text { Transoesophageal echocardiography } & 4(2.4) \\ \text { Cardiac MRI } & \end{array}$


Fig 2. Pie charts showing proportions of cardiac and non-cardiac causes of acute ischaemic stroke and breakdown of cardiac causes of stroke.

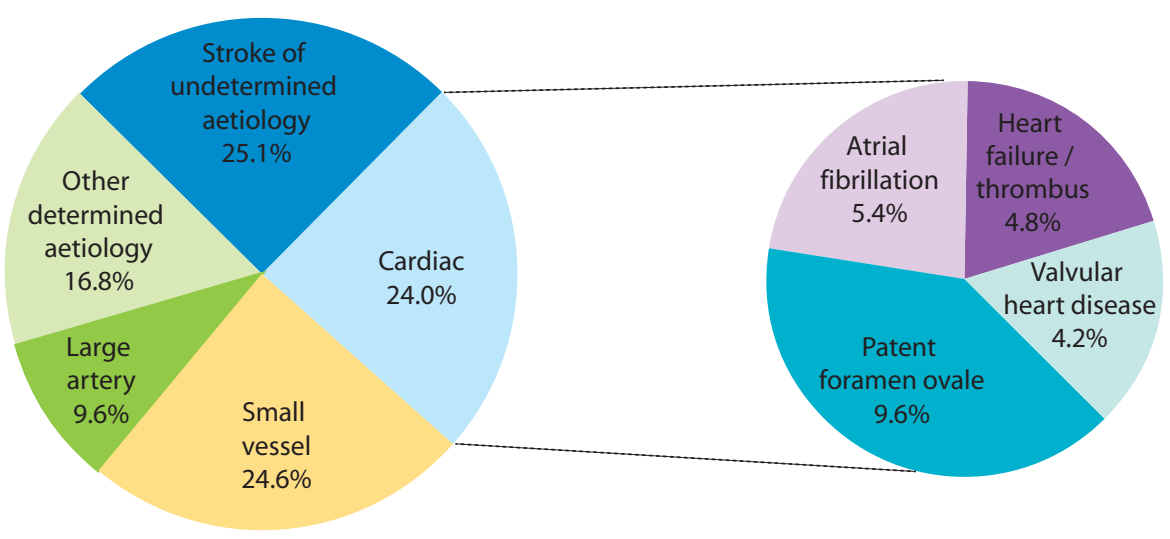

\section{Discussion}

The occurrence of stroke in a young patient can significantly impact on their quality of life. Management is centred on both recovery from the acute episode and also reducing the risk of future events. Identifying the exact cause of the stroke is critically important in facilitating the initiation of specific medical therapy or the consideration of an intervention.

To realise this aim, stroke patients require extensive cardiac and non-cardiac investigations. This would typically include neuroimaging, vascular imaging (of the head and neck vessels), cardiac imaging (TTE) and ambulatory ECG monitoring. If these are negative and it is clinically appropriate, further investigation will include serological testing for rare prothrombotic states. If this is also negative, then bubble contrast echocardiography is performed in order to determine the presence (or absence) of a right-to-left shunt as an occult cause of embolic stroke. If a right-toleft shunt is identified, patients then proceed to transoesophageal echocardiography to confirm the presence of a PFO (or other septal abnormality) and to ensure anatomic suitability for closure. The clinical impression of the treating stroke physician may also guide investigation for other rare causes of stroke.

In our real-world study population, almost one-quarter of strokes were attributable to cardiac causes. This is a significant proportion and justifies the extensive workup that these patients require. It also shows the importance of close collaboration between cardiologists and stroke physicians.

The Sentinel Stroke National Audit Programme (SSNAP) produces national statistics on the characteristics of stroke patients in England, Wales and Northern Ireland. ${ }^{6}$ The AugustNovember 2016 report covering a similar time period to this study detailed that among $>27,000$ patients with stroke, $53.1 \%$ had hypertension, $20.8 \%$ had diabetes mellitus, $26.3 \%$ had prior stroke or TIA and $19.4 \%$ had AF. These data differ from our sample where fewer patients had hypertension $(36.5 \%)$, diabetes mellitus (14.4\%), prior stroke or TIA (12.6\%) or AF $(5.4 \%)$. However, the median age in the national audit was 77 years whereas our population was much younger (median 49 years). It is likely that younger patients presenting with stroke have a range of different causes with a greater preponderance toward cardiac causes such as PFO and non-cardiac causes such as arterial dissection and rare metabolic, genetic and prothrombotic diseases. The trend of increasing stroke incidence in young patients while decreasing in older patients may be explained by these populations having different causes of stroke. Additional contributors could include trends in conventional cardiovascular risk factors and improved primary prevention.?

Ornello et al reported that $22 \%$ of ischaemic strokes were due to cardioembolic causes in a meta-analysis of 65 studies from 1993 to 2015. ${ }^{7}$ This is broadly in agreement with our study. Importantly, this analysis did not include study populations limited to specific age groups and there was significant heterogeneity across the included studies with estimates for the proportion attributable to cardiac causes ranging between $5 \%$ and $44 \%$.

There are only limited data specifically focusing on the incidence of different causes of ischaemic stroke in young people. One prospective study of 394 young patients reported that cardioembolism accounted for $34 \%$, large artery atherothrombosis $12 \%$, arterial dissection $12 \%$ and lacunar stroke $2.5 \% .{ }^{8}$ Our data show fewer with cardiac causes and more with small vessel (lacunar) stroke while the proportions caused by large artery atherosclerosis and arterial dissection are comparable. However, this population was much younger than ours (range 14-47 years) with a much lower prevalence of hypertension and diabetes mellitus.

We acknowledge some limitations of this study. Although we reviewed 3 years of data from a large stroke centre, the overall number of patients is small and these findings warrant confirmation in larger multicentre studies. This was a retrospective study and so is inherently limited by the judgement of reviewers considering the available data. Some data were missing and not every patient screened had every investigation required to identify all possible stroke aetiologies, meaning that a quarter of the cohort could not be categorised with the available data. However, there may be improved external validity with this study design as it reflects actual clinical practice. While it would have been of interest to examine whether investigations were performed on an inpatient or outpatient basis, it was unfortunately not possible to determine this accurately due to the retrospective nature of the study. Some of the patients included in this cohort were managed before the latest current evidence supporting PFO closure and so may not have been investigated with a view to identifying PFO in patients without another aetiology identified. Patients were investigated for AF with ambulatory ECG monitoring with a mean duration of 68.4 hours, however the number of patients diagnosed with AF from this was low. There is evidence that longer periods of monitoring with an implantable device would identify more patients with paroxysmal AF.,10 


\section{Conclusions}

A large proportion (24\%) of acute ischaemic strokes in young patients are attributable to cardiac causes and this justifies thorough cardiac investigation in all patients in this demographic. This requires close interdisciplinary links between cardiologists and stroke physicians. .

\section{References}

1 Kissela BM, Khoury JC, Alwell K et al. Age at stroke: temporal trends in stroke incidence in a large, biracial population. Neurology 2012:79:1781-7.

2 Mas JL, Derumeaux G, Guillon B et al. Patent foramen ovale closure or anticoagulation vs. antiplatelets after stroke. N Engl J Med 2017;377:1011-21.

3 Sondergaard L, Kasner SE, Rhodes JF et al. Patent foramen ovale closure or antiplatelet therapy for cryptogenic stroke. N Engl J Med 2017:377:1033-42.

4 Saver JL, Carroll JD, Thaler DE et al. Long-term outcomes of patent foramen ovale closure or medical therapy after stroke. N Engl J Med 2017:377:1022-32.
5 Adams HPJr, Bendixen BH, Kappelle LJ et al. Classification of subtype of acute ischemic stroke. Definitions for use in a multicenter clinical trial. TOAST. Trial of Org 10172 in Acute Stroke Treatment. Stroke 1993;24:35-41.

6 Royal College of Physicians. Sentinel Stroke National Audit Programme (SSNAP): Clinical audit August-November 2016 public report: National results. London: RCP, 2017.

7 Ornello R, Degan D, Tiseo C et al. Distribution and temporal trends from 1993 to 2015 of ischemic stroke subtypes: a systematic review and meta-analysis. Stroke 2018;49:814-9.

8 Rasura M, Spalloni A, Ferrari M et al. A case series of young stroke in Rome. Eur J Neurol 2006;13:146-52.

9 Sanna T, Diener HC, Passman RS et al. Cryptogenic stroke and underlying atrial fibrillation. N Engl ] Med 2014;370:2478-86.

10 Cotter PE, Martin PJ, Ring L et al. Incidence of atrial fibrillation detected by implantable loop recorders in unexplained stroke. Neurology 2013;80:1546-50.

Address for correspondence: Dr Neil Ruparelia, Royal Berkshire Hospital, London Road, Reading RG1 5AN, UK. Email: neil.ruparelia@nhs.net

\section{Mental health and wellbeing resource}

This new online resource aims to shine a spotlight on the mental health and wellbeing of physicians by opening up the conversation about mental health issues and their impact.

It will help you recognise the warning signs in yourself and others, as well as know about the steps you can take to stay well and seek the right support when you need it.

www.rcplondon.ac.uk/wellbeing

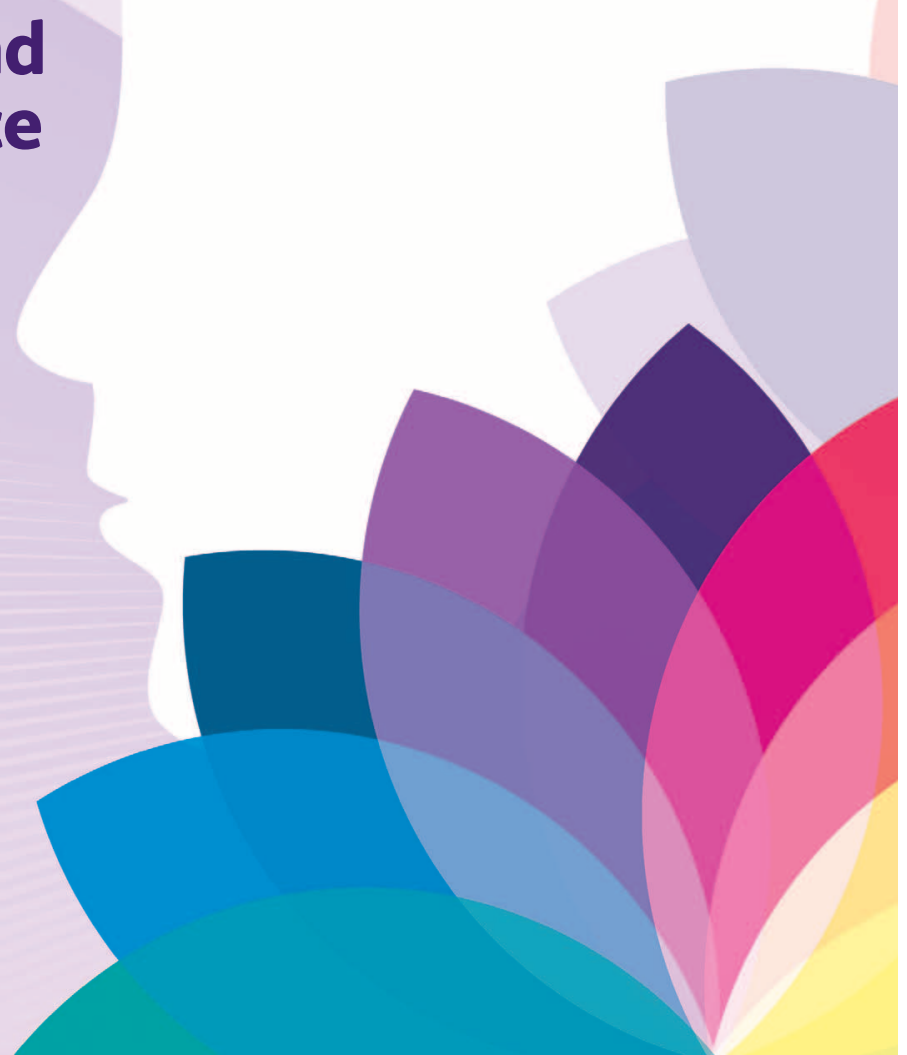

